Archives

$1 \mid 1988$

Varia

\title{
La sidérurgie italienne pendant l'entre-deux guerres et la société des aciéries électriques " cogne »
}

\section{Corrado Binel}

\section{(2) OpenEdition \\ 12 Journals}

\section{Édition électronique}

URL : http://journals.openedition.org/ccrh/2973

DOI : $10.4000 /$ ccrh.2973

ISSN : 1760-7906

Éditeur

Centre de recherches historiques - EHESS

\section{Édition imprimée}

Date de publication : 2 avril 1988

ISSN : 0990-9141

Référence électronique

Corrado Binel, « La sidérurgie italienne pendant l'entre-deux guerres et la société des aciéries électriques « cogne » », Les Cahiers du Centre de Recherches Historiques [En ligne], 1 | 1988, mis en ligne le 13 avril 2009, consulté le 03 mai 2019. URL : http://journals.openedition.org/ccrh/2973 ; DOI : $10.4000 /$ ccrh. 2973

Ce document a été généré automatiquement le 3 mai 2019.

Article L.111-1 du Code de la propriété intellectuelle. 


\title{
La sidérurgie italienne pendant l'entre-deux guerres et la société des aciéries électriques « cogne »
}

\author{
Corrado Binel
}

\section{L'industrie italienne, l'Ansaldo, la guerre et la naissance des aciéries électriques « cogne »}

1 À la mort de Ferdinando Maria Perrone, administrateur délégué et premier actionnaire de Società Anonima Gio, Ansaldo \& C., ses fils Pio et Mario prirent la tête d'un des plus importants groupes industriels italiens ${ }^{1}$.

2 On était alors en 1908, à l'époque où se définit, pour cette entreprise, un vaste projet d'investissement dans le but de s'affranchir des conditions vexatoires imposées à l'industrie mécanique et navale par les grands producteurs sidérurgiques² ${ }^{2}$. En effet, entre 1910 et 1918, prend forme une vaste expérience de diversification de la production, en correspondance avec une phase de croissance dont les chiffres ne donnent qu'une idée purement quantitative ${ }^{3}$.

3 À la même époque, la première Guerre Mondiale, avec ses conséquences sur le marché international, était en train de dévoiler les faiblesses historiques de plusieurs secteurs de l'économie italienne ${ }^{4}$ et particulièrement toutes les contradictions d'une sidérurgie qui était restée hésitante entre un modèle basé sur la refonte de la ferraille et celui du « cycle intégré $»^{5}$.

4 Les industriels de l'électricité, de leur côté, démontraient de plus en plus le rôle clé de la houille blanche dans le développement industriel de la péninsule et ne cessaient d'accroître leur poids économique et politique ${ }^{6}$.

5 L'Ansaldo $^{7}$ se trouvait, donc, comme d'autres entreprises, dans une position peu confortable : d'une part les importations de fonte, d'acier et d'autres produits devenaient de plus en plus ardues et onéreuses à cause de la guerre; de l'autre, la déclaration de 
neutralité de la part de la Suède, qui représentait la tranche majeure des importations du groupe, ne permettait plus d'assurer la continuité productive que les circonstances imposaient.

En même temps, un vaste conflit d'intérêts traversait horizontalement toute la structure industrielle et bancaire du pays, où se livrait une bataille sans merci dans le but explicite d'un réaménagement général redessinant les relations industrielles.

7 L'Ansaldo était à cette époque en première ligne sur tous les fronts, étant en même temps une des plus grandes concentrations industrielles d'Italie et l'un des producteurs majeurs de matériel de guerre. Ce groupe qui, en 1914, comptait 8 usines et employait 12.000 ouvriers, en comptait respectivement 31 et 70.000 en $1919^{8}$. Mais au-delà de la dimension purement quantitative, ce qui est intéressant est le degré de diversification de la production, dans le cadre d'un vaste projet industriel visant à la constitution d'un «système vertical» d'intégration globale (des matières premières aux produits finis) ${ }^{9}$. C'est dans cette conjoncture politique et économique que l'Ansaldo lança le projet industriel qui est au centre de notre analyse, et qui offre l'un des exemples les plus intéressants de l'intégration de l'électro-sidérurgie italienne ${ }^{10}$.

8 Ce projet avait, pour le groupe qui en était le promoteur, plusieurs significations qui, même assez éloignées, constituaient une réponse unique et cohérente à trois grandes questions au moins. Cette ambitieuse réalisation permettait en fait de tenter un affranchissement progressif à l'égard des producteurs d'électricité d'une part, et de l'autre des trusts sidérurgiques qui s'étaient constitués depuis les dernières décennies du siècle précédent, et qui détenaient le monopole de plusieurs productions dans le secteur des constructions navales, mécaniques et d'artillerie. Plus généralement, en vertu du choix opéré, les frères Perrone allaient progressivement compléter leur dessein de «verticalisation » du groupe génois dont ils avaient la responsabilité ${ }^{11}$.

\section{Les mines de fer de Cogne : quelques antécédents}

9 Le 21 janvier 1909 s'était constituée à Gênes la Società Anonima Miniere di Cogne $e^{12}$. Auparavant, et précisément en 1898, la commune de Cogne avait loué les gisements de Liconi et Colonna à Alfred Theys pour un loyer annuel de 3.000 lires, avec la faculté, au cours du bail, d'acheter la mine pour la somme de 80.000 lires. Le 22 décembre 1903, Theys achetait la mine et constituait une société avec le comte Van der Straten Ponthoz, de Bruxelles ${ }^{13}$. Au moment de la constitution de la nouvelle société, Theys et Van der Straten Ponthoz apportèrent de leur côté, comme capital initial, la propriété des mines qu'ils avaient acquises et autres biens pour une somme de 1.200.000 lires soit l'équivalent de 20.000 actions de la société, de façon à en devenir les principaux actionnaires ${ }^{14}$.

Les gisements minéraux furent, à partir de ce moment, l'objet de nombreuses recherches géologiques afin d'en évaluer la consistance totale. Le Corps Royal des Mines déclara l'existence d'un million de tonnes de «bon minerai », l'ingénieur Cortese en 1905 prévoyait 5 millions de tonnes, et l'estimation ultérieure du professeur Vagner fut de 8 millions de tonnes pour l'ensemble des gisements de Liconi et Larcina. Les perforations au diamant effectuées entre 1909 et 1910 par la société suédoise Svenska Diamant Berborrnings Aktienbolaget permirent d'évaluer les seuls gisements de Liconi à plus de 5 millions de tonnes, et les travaux confirmèrent par la suite cette dernière estimation. 
11 La société acheta plus tard les anciennes fonderies et forges d'Aymavilles et Villeneuve, exploitées depuis la première moitié du XIX ${ }^{e}$ siècle et situées dans la vallée principale à quelques dizaines de kilomètres de la ville $\mathrm{d}^{1} \mathrm{Aoste}^{15}$, et démontra dès ses premières interventions sa volonté de dépasser la dimension artisanale qui avait caractérisé jusqu'alors l'exploitation de ces mines. Les techniciens de la société furent employés à l'étude du problème industriel sous tous ses aspects: miniers, sidérurgiques et hydroélectriques, s'inspirant directement de l'expérience suédoise; ce n'est pas un hasard si le premier directeur de la mine fut l'ingénieur suédois Ranjar Nordensten.

Le problème principal à surmonter en vue d'une exploitation rationnelle et économique était et resta pendant plusieurs années celui du transport de la magnétite des $2.500 \mathrm{~m}$ d'altitude où se trouvaient les gisements, à la ville d'Aoste où l'on pensait pouvoir construire les hauts fourneaux pour la réduction du minerai. Plusieurs solutions furent envisagées, dont une prévoyant la construction d'un téléphérique qui aurait dû parcourir la vallée de Cogne dans toute sa longueur ( $20 \mathrm{~km}$ environ) ou traverser les contreforts montagneux qui séparent la mine de la vallée principale d'Aoste; on en vint même à imaginer la construction d'une conduite forcée dans laquelle l'eau et le minerai auraient pu ensemble rejoindre leur lieu de destination. Ce n'est que quelques années plus tard, lors d'une visite des frères Perrone, que l'on projeta la solution qui fut adoptée par la suite : la construction d'une galerie ferroviaire sous le col du Drinc, intégrée à un système de téléphériques.

Les travaux commencèrent dans l'été 1916 pour se terminer avec l'inauguration du tunnel le 18 octobre $1922^{16}$. Dans l'ensemble, les autres infrastructures : téléphériques, centrale électrique, compresseurs, logements pour les ouvriers, ateliers mécaniques et de réparation, étaient déjà terminés à la fin de 1919. La même année, à Cogne, était entrée en fonction l'installation de broyage, de lavage et de séparation magnétique du minerai. On voit donc se préciser entre 1915 et 1919 les deux grandes questions qui justifiaient le projet industriel de l'Ansaldo en vallée d'Aoste : l'exploitation du bassin minier de Cogne et celle des forces hydrauliques de la vallée de la Doire au pied du Mont Blanc ${ }^{17}$.

\section{Crise et restructuration du groupe Ansaldo}

14 En 1921, la crise de la Banca Italiana di Sconto (BIS) entraîna l'Ansaldo dans une période de restructuration profonde et complexe. C'est à ce moment que l'avenir du groupe industriel intéressa encore plus vivement d'autres groupes dont l'ILVA ${ }^{18}$, la F.I.A.T. (Fabbrica Italiana Automobili Torino) et autres qui voyaient dans le développement "vertical» de l'Ansaldo une inquiétante perspective. Effectivement, «une fois que les mines de Cogne, les hauts fourneaux et les installations hydro-électriques valdôtaines auraient été coordonnées en un cycle intégré avec les aciéries de Cornigliano, les usines mécaniques, les chantiers et les compagnies de navigation gênoises, l'Ansaldo se serait transformée (...) en une puissance dominant tous les autres groupes $»^{19}$.

En particulier, cette concentration verticale dont la conséquence était une autonomie de production toujours plus importante, faisait du groupe génois un élément d'un poids déterminant dans le secteur mécanique et sidérurgique, et les accrochages avec l'ILVA, la $T E R N^{20}$, la F.I.A.T. et les banques liées à ces groupes industriels : Credito Italiano et Banca Commerciale, qui avaient caractérisé les années 1910, laissèrent la place à une intervention directe dans la réorganisation de l'Ansaldo de la part d'industriels tels que Odero ${ }^{21}$, Bocciardo $^{22}$ et autres, présents en force dans la sidérurgie et les constructions navales, et 
qui se trouvaient affronter la conjoncture du premier après-guerre non sans difficultés ${ }^{23}$. La F.I.A.T. tout particulièrement, avait des raisons de s'intéresser de près à l'avenir de l' Ansaldo; en effet, ces entreprises étaient fortement présentes dans plusieurs secteurs : la sidérurgie, l'industrie automobile et aéronautique, les machines agricoles et les transports en commun; ce n'était pas seulement l'affrontement de deux producteurs, mais aussi de deux politiques d'entreprise, sinon de deux modèles industriels. Pour Valerio Castronovo, le conflit était «entre une agrégation toujours plus lourde d'une masse de coûts improductifs et d'éléments de spéculation sur la suprématie financière et commerciale, qui semblait résumer de façon particulièrement aiguë, le décalage entre un système rigide et hétérogène, caractérisé par un faible rapport entre chiffre d'affaires et immobilisations (...) et un système industriel, au contraire, en phase de renouvellement profond des techniques et de l'organisation du travail, beaucoup plus attentif aux coûts de production et aux marchés $»^{24}$.

En mars 1922, Bonaldo Stringher, directeur général de la Banca d'Italia, convoqua d'urgence le Credito Italiano et la Banca commerciale afin d'obtenir les financements nécessaires au plan de restructuration de l'Ansaldo ${ }^{25}$. C'est après le refus de ces banques de s'engager directement dans l'opération, que prit corps la décision de s'appuyer sur la section autonome du Consorzio Sovvenzioni su Valori Industriali (CSVI), c'est-à-dire sur un financement public; l'intervention de l'Etat dans l'économie prend ainsi une nouvelle tournure, pour arriver à son point culminant en 1933 avec la constitution de l'I.R.I. (Istituto per la Ricostruzione Industriale) ${ }^{26}$.

17 L'intervention de Stringher, mais surtout celle du Président du Conseil Benito Mussolini, seront donc à l'origine des nouvelles sociétés Ansaldo S.A. et Ansaldo-Cogne ${ }^{27}$, cette dernière ayant la gestion des mines et des installations hydro-électriques et sidérurgiques de la vallée d'Aoste; mais les rapports entre les différents groupes du pouvoir économique, industriel et financier, et leur rôle dans les années de la "Marcia su Roma ", les nuances dans leur soutien au gouvernement Mussolini sont un autre élément qui a sûrement joué un rôle déterminant dans la réorganisation du plus grand groupe industriel de la péninsule ${ }^{28}$.

\section{Ansaldo-Cogne et Cogne-Girod : les premiers aciers spéciaux}

La société Ansaldo-Cogne avait à son actif, au moment de sa constitution, une importante réserve de capitaux liquides, ce qui explique les importants investissements des années 1922-1924, la mise en marche des hauts fourneaux électriques suédois Electrometal, de l'aciérie, des fours Héroult, du laminoir, et c'est presque une crise de croissance s'ajoutant aux difficultés de maîtrise des nouvelles technologies de l'électro-sidérurgie, qui expliquent par contre l'arrivée à la direction du secteur acier de l'Ansaldo-Cogne de l'ingénieur Paul Girod ${ }^{29}$, fondateur des Aciéries électriques Paul Girod d'Ugine (Savoie) ${ }^{30}$.

En 1924, la collaboration technique de Paul Girod donnera lieu à la constitution d'une nouvelle société sous la raison sociale Cogne-Girod, au capital social de 20.000.000 de lires, composé à 50 \% par l'apport de la part de l'Ansaldo-Cogne "secteur aciers ", et à 50 \% par l'apport financier du "groupe suisse » composé par la Banque Suisse, Jules Block, Georges Stadaler et Paul Girod lui-même ${ }^{31}$. Sous la direction de Paul Girod, l'aciérie connut alors un essor considérable, grâce à la nouvelle organisation du laminoir et à l'installation dans le secteur des traitements thermiques de fours Stein alimentés par 4 gazogènes Kerpely ${ }^{32}$. Les orientations industrielles des années 1923-1926 montrent en même temps la relance, 
comme au temps des frères Perrone, de la "centralité » de la production hydroélectrique. Elle figure l'élément central et moteur de l'expansion du secteur sidérurgique. En deuxième lieu, l'apport technique et financier du "groupe suisse», mais aussi la recherche volontaire de cette collaboration, mettent en évidence de nouvelles orientations industrielles. Bien sûr, les frères Perrone les premiers avaient songé aux produits spéciaux dans le secteur de la fonte et des aciers, mais les possibilités pratiques de réalisation, techniques et financières, avaient jusqu'alors fait défaut.

En réalité, si la production des aciers spéciaux devient à partir de la moitié des années 1920 une spécialité du groupe valdôtain, par contre la production de fonte de qualité dans les hauts fourneaux électriques ne trouva jamais une véritable solution technique. A l'origine de cet échec: le minerai, ou mieux sa gangue particulièrement dure, les difficultés d'approvisionnement en combustible adéquat, et les problèmes de mise au point des hauts fourneaux. On peut ainsi affirmer que, du fait de la discontinuité qualitative du produit et d'autres inconvénients techniques, la production de «fonte électrique » n'assuma jamais un véritable caractère industriel, défini par la continuité de la production et la constance de la qualité.

La société Cogne-Girod, en utilisant de la ferraille plus que de la fonte produite sur place, et en supportant de ce fait des frais très élevés par rapport à d'autres usines mieux placées géographiquement, réussit à conquérir une part relativement importante du marché national. A la fin de l'année 1927, la société Ansaldo-Cogne, sans doute poussée par le gouvernement italien, ayant épuisé ses liquidités, obtint du Ministère du Trésor un crédit de 55 millions de lires pour couvrir les frais de sa participation croissante dans la société Cogne-Girod jusqu'à sa définitive «italianisation » : opération fort probablement justifiée par la place de plus en plus centrale occupée par la sidérurgie spéciale de guerre» dans la politique de réarmement qui caractérisera les années 1930. Après l'exclusion de Paul Girod, et la transformation de l'Ansaldo-Cogne en Società Anonima Nazionale Cogne en 1929, s'affirme un programme industriel qui en moins de deux ans va complètement bouleverser la physionomie du groupe sidérurgique valdotain.

Seront en effet mis en oeuvre : deux hauts fourneaux à coke; une aciérie Bessemer en duplex avec 4 fours Héroult de 20 tonnes, 1 four Tagliaferri de 5 tonnes, un four Secomet de 5 tonnes et un four Bassanese d'une tonne ; un laminoir pour fers longs avec blooming de $1.000 \mathrm{~mm}$. et trains de laminage jusqu'à $5 \mathrm{~mm}$. de diamètre pour les profilés cylindriques et $4 \mathrm{~mm}$ pour les fers carrés; mais surtout la puissance globale des installations électriques passera de 12.000 à $50.000 \mathrm{kwh}$, pour une capacité productive annuelle d'environ 200 millions de kwh. Enfin sera mise en activité la mine de charbon de La Thuile, et pour son exploitation sera construit un chemin de fer de 30 kilomètres, d'Aoste jusqu'au pied du Mont Blanc. L'endettement de la société croîtra de manière vertigineuse à la fin des années vingt, et la crise économique, qui débute en 1929, ouvrira une époque d'incertitudes qui durera jusque vers $1935^{33}$.

\section{Les années trente : la « sidérurgie spéciale de guerre » et le rôle de l'Etat}

En 1933, l'I.R.I. lança une étude sur la situation économique, productive et patrimoniale des H.S.A. Cogne et Aosta ${ }^{34}$. Donato Menichella, alors président de l'I.R.I., en confia la direction à l'ingénieur Agostino Rocca qui fut, avec Oscar Siniglia et quelques autres, l'un des plus importants dirigeants de la sidérurgie du secteur public en Italie durant les 
quinze dernières années du régime fasciste. L'étude donna lieu à un important rapport sur le groupe électro-sidérurgique valdotain, et sur les différentes possibilités de réorganisation $\mathrm{du}$ groupe dans le cadre d'une révision générale de la politique sidérurgique italienne. Grâce au travail coordonné par Agostino Rocca, une première réflexion est aujourd'hui possible sur l'histoire tourmentée de ce complexe minier et sidérurgique.

Au préalable, rappelons qu'au début des années 1930, et particulièrement en 1933, on constate une forte progression des études concernant l'état de santé et les perspectives de la sidérurgie italienne. L'ILVA émerge à cette époque comme une entreprise de toute première importance grâce à son rôle de principal producteur national d'aciers ordinaires et parce qu'elle est, avec un petit nombre d'entreprises, un producteur au moins partiellement intégré ${ }^{35}$. Mais, à la même époque, la "sidérurgie spéciale de guerre ${ }^{36}$ semble faire son chemin propre, en tant que secteur de plus en plus essentiel pour le régime compte tenu, en particulier, de ses perspectives d'expansion coloniale. Dans ce secteur de la défense nationale, trois sociétés passent au premier plan : la Terni, l' Ansaldo et la $\operatorname{Cogne}^{37}$. Les deux dernières sont déjà, depuis la crise de 1921, largement contrôlées par l'Etat à travers le Ministère des Finances, et la prise de contrôle de la Terni par l'I.R.I., «conséquence des opérations d'assainissement bancaire, détermina une situation qui plaçait les trois entreprises italiennes opérant dans le secteur de la sidérurgie spéciale de guerre (...) sous le contrôle direct de l'Etat, et rendit donc possible leur assujettissement à une gestion unique $»^{38}$.

C'est d'ailleurs précisément dans cette direction que la commission Parravano semble vouloir opérer; on peut en effet lire dans son rapport: «Parvenus à ce point de notre travail, on nous a suggéré une heureuse directive qui se propose d'impliquer également, dans l'étude de cet arrangement (de la sidérurgie spéciale de guerre), d'autres secteurs de l'IRI, dans le but de trouver la solution qui non seulement sera la plus favorable à la Cogne, mais répondra le mieux possible aux intérêts supérieurs de l'économie nationale. On attire en particulier notre attention sur l'éventualité de concilier les intérêts de la Cogne avec ceux de l'Ansaldo, dont l'organisation reste un problème à résoudre ${ }^{39}$. On voit émerger cette volonté de bipolarisation du secteur, qui semble avoir rencontré la faveur de l'administrateur délégué de la Terni, Arturo Bocciardo, dont l'action eut certainement une influence déterminante grâce à ses contacts directs avec le chef du gouvernement. En effet ce choix, par lequel on envisageait la constitution d'un pôle unique Ansaldo-Cogne, permettait en même temps à la Terni de conserver une autonomie industrielle considérable. Le projet de fusion fut donc soumis à Mussolini; en dépit de son soutien déclaré, l'opération rencontra encore de nombreuses difficultés sur son chemin, dues aux résistances, d'un certain point de vue parfaitement justifiées, que les deux sociétés manifestèrent à la cession de leurs installations. Le 21 septembre 1934, la nouvelle société démarra son activité sous la raison sociale de Società Italiana Acciaierie Cornigliano-Cogne (S.I.A.C.C.), au capital social de 50 millions de lires, entièrement souscrit par l'I.R.I. ${ }^{40}$.

\section{L'expérience de la S.I.A.C.C. : septembre 1934 - juin 1935}

La réorganisation de ce segment de la sidérurgie italienne tournait autour des rapports entre les trois entreprises, et on en vint à la conclusion que la sidérurgie spéciale de guerre devait être concentrée en deux pôles: Terni et Gênes. Ce choix aurait permis de concentrer à Cornigliano un vaste complexe industriel pour la production des aciers, 
tandis qu'en vallée d'Aoste restaient en place les hauts fourneaux et tout le système minier et hydro-électrique. Cette décision fut certes influencée par des raisons économiques et militaires, mais fort probablement aussi par les aspirations personnelles d'Agostino Rocca, manager ambitieux et subtil, qui entrevoyait la possibilité de parvenir à la direction d'un groupe imposant et compétitif. Pour des raisons difficiles à cerner, Agostino Rocca ne joua qu'un rôle de second plan dans la nouvelle société, tandis qu'un an plus tard, à la faillite de l'expérience de fusion Ansaldo-Cogne, c'est-à-dire au moment de la constitution de la Società Italiana delle Acciaierie di Cornigliano (S.I.A.C.), ce fut précisément Agostino Rocca qui fut élevé à la plus haute responsabilité.

La société S.I.A.C.C. avait été créée dans le but de « remplacer (dans le secteur des aciers spéciaux) les importateurs étrangers : Krupp, Boher, Ugine, Holzer, Alpine, etc. $\aleph^{41}$ et de provoquer une concentration industrielle publique et plus précisément I.R.I., face aux initiatives des industriels privés sur le marché national. Dans une lettre de Rocca de janvier 1935 on peut lire : «Les Acciaierie e Ferriere Lombarde ont préparé un projet d'usine pour aciers spéciaux que l'on installerait à Bolzano, et il semble que cette aciérie soit désirée par les autorités supérieures. De plus, on a accordé récemment un permis de construire pour les installations d'une petite société dirigée par le sénateur Brezzi, qui a l'intention de produire l'alliage "Widia $»^{42}$ en Italie. En outre, les autres producteurs : Breda et FIAT, intensifient leur production. La S.I.A.C.C. veut affirmer la sienne grâce à des qualités techniques et d'organisation, et ne demande pas de mesures de protection particulières comme l'a fait la Cogne. Mis à part les intérêts de la S.I.A.C.C. on peut considérer comme absurde de permettre à d'autres organismes de production de se lancer sur un marché aussi petit et limité, quand il y a déjà une firme, la S.I.A.C.C., qui suffit largement, et qui a été précisément créée dans ce but, au prix de très gros sacrifices de la part de l'État ${ }^{43}$. Sacrifices financiers de l'Etat, mais aussi des employés, des dirigeants et surtout des milliers d'ouvriers qui risquèrent à cette époque de se retrouver face à l'alternative : chômage ou émigration.

Il est en effet difficile de dire dans quelle mesure pesèrent les facteurs humains, et donc ceux du consensus, dans la décision de Mussolini de mettre un terme à l'aventure de la S.I.A.C.C. ; mais les dizaines et les dizaines de lettres de remerciement et de dévotion à la " clairvoyance du Duce », envoyées pour l'occasion par le Podestà d'Aoste, par le Préfet de la province et par de nombreuses personnalités en portent un témoignage indirect ${ }^{44}$.

29 À la nouvelle société, l'Ansaldo devait apporter l'ensemble de l'aciérie de CorniglianoCampi ; la Cogne, quant à elle, devait apporter les mines de fer de Cogne et toutes les installations sidérurgiques valdotaines. La Società Anonima Nazionale Cogne conservait la participation dans la Società Anonima Nazionale Aosta-Impianti Elettrici, dont la situation serait réglée à part, compte tenu soit des résultats de l'étude de l'I.R.I., soit des nécessité énergétiques des aciéries de Cornigliano ; elle gardait également les mines de houille de La Thuile et l'ensemble des constructions civiles et des terrains non industriels situés en vallée d'Aoste.

En réalité, la situation n'était pas facile à résoudre, ne serait-ce que du point de vue patrimonial ; cependant d'autres problèmes grevèrent le départ de la société, ainsi que la faillite précoce de l'expérience S.I.A.C.C., en particulier des problèmes techniques, liés au transfert du laminoir et de la presse Schloemann pour la fabrication des corps des canons, et des problèmes de transfert de personnel. Les dirigeants et les techniciens devaient être licenciés et réembauchés à l'essai en attendant la définition du nouvel organigramme de l'entreprise: la mise en congé de toute la couche supérieure du personnel aurait 
comporté, entre autres, des frais très lourds surtout pour l'Ansaldo, car à Gênes des coutumes locales très rigides étaient en vigueur en matière de préavis et d'indemnités de licenciement ; mais, de façon plus générale, avec la constitution de la S.I.A.C.C., la Cogne entrait de fait en liquidation, et pour ses 4.000 ouvriers s'ouvrait une période de forte incertitude.

31 Sur le plan national, la naissance de la S.I.A.C.C. posait de nouvelles questions tant au niveau des relations inter-industrielles qu'à celui des équilibres du marché; il était en particulier évident que la nouvelle société ne pouvait pas ne pas définir sa propre position par rapport, au moins, à la Terni, c'est-à-dire l'autre pôle du projet de réorganisation de la sidérurgie spéciale de guerre. Jusqu'au mois de mai 1935, date qui marque l'échec définitif de la S.I.A.C.C, et même en tenant compte des «intérêts supérieurs de la Nation ", on ne parvint jamais à un accord avec la Terni, et ceci malgré le fait que les deux parties étaient apparemment disposées à prendre en considération le schéma d'accord déjà préparé par Arturo Bocciardo, administrateur délégué de la Terni ${ }^{45}$.

\section{Des guerres coloniales à la guerre mondiale, ou l'époque de l'expansion}

C'est donc, comme on le voit, de crise en réorganisation que l'on arrive au milieu des années trente, c'est-à-dire à la ligne de départ d'une époque d'expansion qui amènera le groupe à compter en 1944 environ 10.000 ouvriers. Cette époque de croissance correspond aussi à un changement important dans la politique économique du régime ${ }^{46}$. C'est l'époque de la conversion à l'autarcie, des grands travaux publics, de la conquête en Afrique orientale d'une «place au soleil». C'est l'époque du plus haut niveau de consensus au régime mussolinien, et, en même temps, du plus rigide contrôle policier sur les masses populaires.

L'autarcie sidérurgique se développa dans le sens de l'augmentation de la production hydro-électrique et dans celui des recherches minières capables de réduire la dépendance à l'égard des importations de matières premières. Pour élever l'entreprise à « l'électrosidérurgie pure ", on décida la construction d'une nouvelle centrale à La Salle, dans la haute vallée d'Aoste, et l'achèvement des installations de Chavonne (commune de Villeneuve). Pour la réalisation de ces travaux, on ouvrit en 1937 un crédit de 60 millions de lires auprès de l'Istituto Mobiliare Italiano (I.M.I.). On effectua, au même moment, des améliorations dans les ateliers de ferro-alliages, et le nouvel établissement mécanique d'Imola (province de Bologne) fut lancé.

La recherche de minéraux ferreux, parallèlement à celle que menait la Finsider, fut particulièrement active. Venant s'ajouter aux forages en profondeur effectués dans la mine de Cogne, on inaugura un important programme de sondages en Sardaigne, dans la zone de recherche de Giacuro, et après une période d'essai, on acheva une petite installation pilote à Neptune, près de Rome, pour l'extraction de la magnétite des sables ferrugineux du littoral. En 1940, la première mine de Gonnosfanadiga, en Sardaigne méridionale, commença à extraire le molybdène. La flambée productive est prouvée par les chiffres : le chiffre d'affaires, de 82 millions en 1934, passe à 569 millions en 1941, et le 31 mars 1942 le capital social passe de 250 à 400 millions de lires ${ }^{47}$.

Le plan du général Pariani pour le renouvellement des artilleries de l'armée, tout comme d'autres commandes (des chemins de fer nationaux, de l'aviation militaire et de la marine 
de guerre), firent sentir leurs effets dès 1937-38 ; mais dès 1936, à l'engagement italien en Abyssinie et en Espagne correspondit une croissance importante sur le graphique des productions. Les véritables productions militaires, réparties dès le début des années 40 entre l'Ansaldo et l'O.T.O., arrivèrent également à Aoste, grâce à la " haute intercession » du Ministre des Finances Paolo Thaon de Revel, qui avait déjà eu un rôle de premier plan lorsqu'en 1935, il s'était opposé à «l'irisation » de l'entreprise valdotaine. Mais lorsqu'en 1942, l'ingénieur Alberto Bettica devint administrateur délégué de la Cogne, ce fut le début d'un jeu difficile contre les visées de la FIAT., Valletta ${ }^{48}$ tenta, en effet, dans les derniers mois de 1942, de s'assurer le contrôle des aciéries valdotaines. Bettica écrivait à ce propos à son ami Paolo Thaon de Revel: «Lui ayant objecté qu'il lui fallait l'approbation du Ministre des Finances, Valletta me répondit textuellement : approbation ou non, je vais voir le Duce, et s'il ne nous donne pas la Cogne, il ajoutera une autre grande responsabilité à celles qu'il a déjà prises. Il est temps d'en finir $! »^{49}$. Et, de fait, Valletta obtint l'autorisation pour une participation de $20 \%$ dans la S.A.N. Cogne.

L'administrateur délégué s'opposa encore avec force à une participation si importante du capital privé, étant d'avis que « la Cogne a une organisation et des visées très différentes de celles de la FIAT, société à caractère spéculatif, qui touche de nombreux secteurs de l'activité industrielle et se fonde essentiellement sur les valeurs de bourse $»^{50}$. Dans une lettre de Bettica on peut encore lire : "Valletta n'a pas pensé que le doublement de ses Ferriere Piemontesi peut égement se faire dans les Ateliers du Rhône (...) En ce qui me concerne, je préférerais redoubler la Cogne à Saint-Etienne plutôt qu'à Courgné ${ }^{51}$. L'assaut manqué de la Cogne tenté par la FIAT avait plus d'une raison d'être. Valletta aurait fait d'une pierre au moins trois coups: contrôler une entreprise productrice d'aciers spéciaux, qui était à la fois très utile et concurrente des Ferriere Piemontesi dans certaines productions ; diminuer l'impact, sur tout le cycle productif, du bombardement possible, précisément dans ces mois-là, des établissements sidérurgiques du groupe turinois ; et en dernier lieu, en raison de son rôle de réserve financière potentielle, qui lui venait $d^{\prime}$ '«immobilisations colossales en demi-produits et des crédits considérables accordés $»^{52}$, l'achat de la Cogne aurait été, de toute façon, une affaire particulièrement intéressante.

\section{Le développement de l'entreprise dans le contexte de la sidérurgie italienne des années vingt et des premières années trente}

37 L'histoire de « notre » entreprise nous amène à une réflexion conduite, pour ainsi dire, de l'intérieur, sur un certain nombre de questions, ou plutôt d'interprétations du développement du secteur sidérurgique italien pendant l'entre deux guerres. Plusieurs éléments doivent en effet être pris en compte pour expliquer, dans sa dimension macroéconomique, l'évolution de ce secteur.

Un premier élément est celui des relations économiques internationales qui connaissent, à cette époque, une période d'instabilité et de mutation. Plus précisément, dans le secteur du fer et de l'acier, on remarque un excès de la capacité de production, qui s'accompagne d'une forte instabilité des prix au sein d'une tendance fondamentalement négative. Dans cette situation, la structure productive italienne ne peut que révéler toutes ses faiblesses face à une concurrence internationale renforcée sur les marchés européens.

Une deuxième question, liée encore à la situation internationale, est celle de la tendance à utiliser toujours plus largement la ferraille dans la production des aciers. Cette tendance 
était alimentée par la disponibilité accrue de cette matière première, liée à la reconversion de la production dans l'après guerre.

En deuxième lieu, dans ce contexte d'instabilité, les entreprises s'orientent vers des cycles productifs d'une plus grande souplesse, tels celui de la refonte de la ferraille. Dans le cas italien, aux éléments qu'on vient de citer, et qui militent en faveur d'une "sidérurgie de la ferraille », viennent s'ajouter d'autres considérations, particulièrement de nature idéologique, produits d'une culture libérale qui dénie à ce pays, dépourvu d'importantes ressources naturelles, la possibilité historique de parvenir à constituer une véritable industrie du fer et de l'acier. Toujours sur le plan de la politique économique intérieure, les modifications imposées en 1923 aux tarifs sur les importations des fontes et des ferrailles montrent de façon explicite une volonté de destructuration de la sidérurgie de la fonte et du « cycle intégré ».

41 Un tel contexte, qui n'explique pas à lui seul les difficultés des sociétés telles que l' Ansaldo-Cogne ou la Cogne-Girod dans cette décennie, nous met en présence des obstacles que ces entreprises ont dû essayer de surmonter, au-delà de ceux que suscitaient le marché ou le cadre des relations inter-industrielles. On sait en fait que le marché intérieur évolue très lentement : en 1938, par exemple, la consommation italienne d'acier par tête est la moitié de la consommation française, le quart de l'anglaise, le sixième de l'américaine. Mais on sait aussi, plus généralement, que la réponse stratégique de la grande industrie mécanique et sidérurgique aux crises de croissance du début du $\mathrm{xx}^{\mathrm{e}}$ siècle avait été celle de l'intégration des cycles productifs, des matières premières aux produits finis, comme le montre l'expérience de l'Ansaldo ou celle de la FIAT.

Ces entreprises n'expriment donc pas la réalité des conditions historiques de l'économie et du marché, et affrontent leur avenir dans des conditions paradoxales. Le passage massif des entreprises sidérurgiques italiennes, entre le début des années vingt et le début des années trente, au secteur public est-il la conséquence d'un désir croissant de contrôle des secteurs qui sont au centre d'une économie de guerre? S'agit-il d'arracher à la libre concurrence certains secteurs producteurs de matières premières afin de créer un système d'aide indirecte au secteur privé, ou encore, le prix de la crise du premier après guerre, des spéculations, des affrontements entre groupes du pouvoir politique et économique, a-t-il été la création d'entreprises défiant les logiques propres d'une économie de marché ?

Tous ces éléments ont sûrement joué simultanément, mais s'il était possible d'accéder aux archives de l'entreprise ${ }^{53}$, pour procéder à une analyse de sa politique industrielle, on pourrait relativiser le poids de chacune de ces hypothèses. Comme on l'a vu, les années 1935-1944 représentent pour l'entreprise un vrai tournant. Ce tournant correspond aussi à l'arrivée sur le marché d'un nouveau client, c'est-à-dire l'Etat. Encore une fois, ce n'est donc pas une bataille gagnée face à la concurrence et au marché qui a assuré une décennie de croissance et de bénéfices ; c'est plutôt un mécanisme qui s'est mis en place à la suite de la crise des années 1929-1933 et qui trouve finalement l'impulsion nécessaire à son développement. La Società Nazionale Cogne devient alors, plus qu'une entreprise nationalisée, une entreprise d'Etat. Mais encore une fois les papiers conservés par la société deviennent d'une importance capitale pour comprendre si, dans cette conjoncture, une entreprise qui a toujours affiché, et avec orgueil son autonomie, est encore en mesure de développer une politique industrielle propre, et à quel niveau d'indépendance par rapport au pouvoir politique. 


\section{Remarques sur la formation de la classe ouvrière du groupe minier et électro-sidérurgique cogne}

d'embauche $\aleph^{54}$ des ouvriers, des origines de l'entreprise (1917) aux années 50, dans tous les secteurs miniers et sidérurgiques ${ }^{55}$. Le travail est fondé sur un échantillon au $1 / 25$ e, soit 1.475 cas pris en considération sur 36.836 ouvriers embauchés ${ }^{56}$. On a donc pu travailler sur une masse relativement considérable de données, qui ont été organisées autour d'un certain nombre de questions : niveaux d'emploi, mobilité professionnelle ou turn-over, qualification ou encore provenance géographique de la main d'oeuvre. Ce travail s'insère dans une tendance plus générale du champ de recherche de l'histoire économique italienne, qui connaît depuis le début des années 80 un dynamisme prometteur. Auparavant, au début des années 60 , tout comme dans le courant des années 70 , l'intérêt des sociologues comme des économistes, des partis comme des organisations syndicales, s'était concentré sur un modèle, sur un type particulier de travailleur, représenté par l'ouvrier industriel à poste fixe et ayant assez souvent une haute professionnalité, en vertu du rôle politique que ce segment de classe ouvrière était en train de jouer dans les conflits politiques et sociaux de l'Italie du second après-guerre ${ }^{57}$. Ces dernières années, l'ensemble de la recherche semble s'ouvrir à de nouvelles hypothèses, et le monde du travail industriel est de plus en plus l'objet d'une réflexion autour de questions telles que la mobilité professionnelle et géographique de la classe ouvrière, ses stratifications, ses stratégies de comportement dans la structure sociale et productive; en d'autres termes l'intérêt se déplace de plus en plus vers l'étude de la complexité et de la multiplicité des comportements politiques et sociaux du monde du travail, plutôt que sur son rôle de transformation structurelle de la sociétés8. originaux du développement d'un milieu ouvrier défini sur la base de l'analyse des sources qui sont une part du patrimoine des entreprises elles-mêmes, telles que les «livres d'embauche» (plutôt que les dossiers individuels du personnel) ${ }^{59}$. Ce type d'enquête, menée à partir des entreprises sur un territoire déterminé, permet de sortir des impasses de la «macro-histoire» pour pénétrer dans une sorte d'histoire territorialisée, qui n'est pas de l'histoire locale, mais plutôt la délimitation de l'espace de confrontation entre différentes hypothèses de lecture des modes de transformation des structures économiques et sociales. C'est dans cette dimension que certaines relations apparaissent comme des éléments réels d'explication : par exemple, entre monde rural et travail d'usine, dont les relations apparaissent inséparables de la trame épaisse des relations sociales dans le monde rural; en dernière analyse, l'usine n'apparaît plus comme une "rupture ", comme un élément à étudier séparément, mais comme une composante d'un tout à prendre en considération dans son ensemble ${ }^{60}$.

En ce qui concerne notre travail, il faut préciser que nous avons pu nous servir des séries historiques complètes et homogènes, intégrées par les "dossiers individuels » qui permettent une reconstruction minutieuse des carrières professionnelles de chacun. Les données qui suivent sont regroupées autour de trois critères principaux: provenance géographique de la main d'oeuvre; qualification ouvrière ; durée des rapports de travail ${ }^{61}$ . En ce qui concerne la provenance régionale des travailleurs, les données sont les suivantes :

Les Cahiers du Centre de Recherches Historiques, 1 | 1988 
Tableau $\mathrm{n}^{\circ} 1$ (données par régions administratives relatives aux seuls établissements sidérurgiques d'Aoste pour les années 1922 et 1942).

\begin{tabular}{|c|c|c|}
\hline Abruarias & $1,2 \%$ & 0,89 \\
\hline Ca labre & $1,2 \%$ & $6,8:$ \\
\hline Campanies & 1,28 & $0,8:$ \\
\hline Emilie eto Romagne & $2.5 \%$ & $2.5 \%$ \\
\hline Etranger & $0,0 \%$ & $2,9 ?$ \\
\hline Frioul & $6,0 \%$ & $0,8:$ \\
\hline Ligure & $1,2 \%$ & $1,2: 8$ \\
\hline Lombanrd ie & $4,9 \%$ & $2,9: 4$ \\
\hline Piemont: & $16,8 \%$ & $42,3: 4$ \\
\hline Poutilles & $1.2 \%$ & $0,0: 4$ \\
\hline Sardatignes & $1,2 \%$ & $0,0:$ \\
\hline sicile & $0,0 \%$ & $1,2:$ \\
\hline Toscane & 1,28 & $1,2:$ \\
\hline Trent:in et: sud Tyrol & $1,2 \%$ & $0.4: 8$ \\
\hline Ornbinis & $1,2 \%$ & $0,4: 8$ \\
\hline Vallee d'Aoste & $49,4: \%$ & $43,8: 4$ \\
\hline Vẻnę̆t: fie: & $9,6 \%$ & $22,0: 4$ \\
\hline Autress rëgions & $0,0 \%$ & $0,0:$ \\
\hline
\end{tabular}


Tableau $\mathrm{n}^{\circ} 2$ Provenance par grands ensembles régionaux de la main d'oeuvre des établissements sidérurgiques, des mines de Cogne (Fer) et des mines de La Thuile (houille) (moyenne des données de 1922 à 1942)

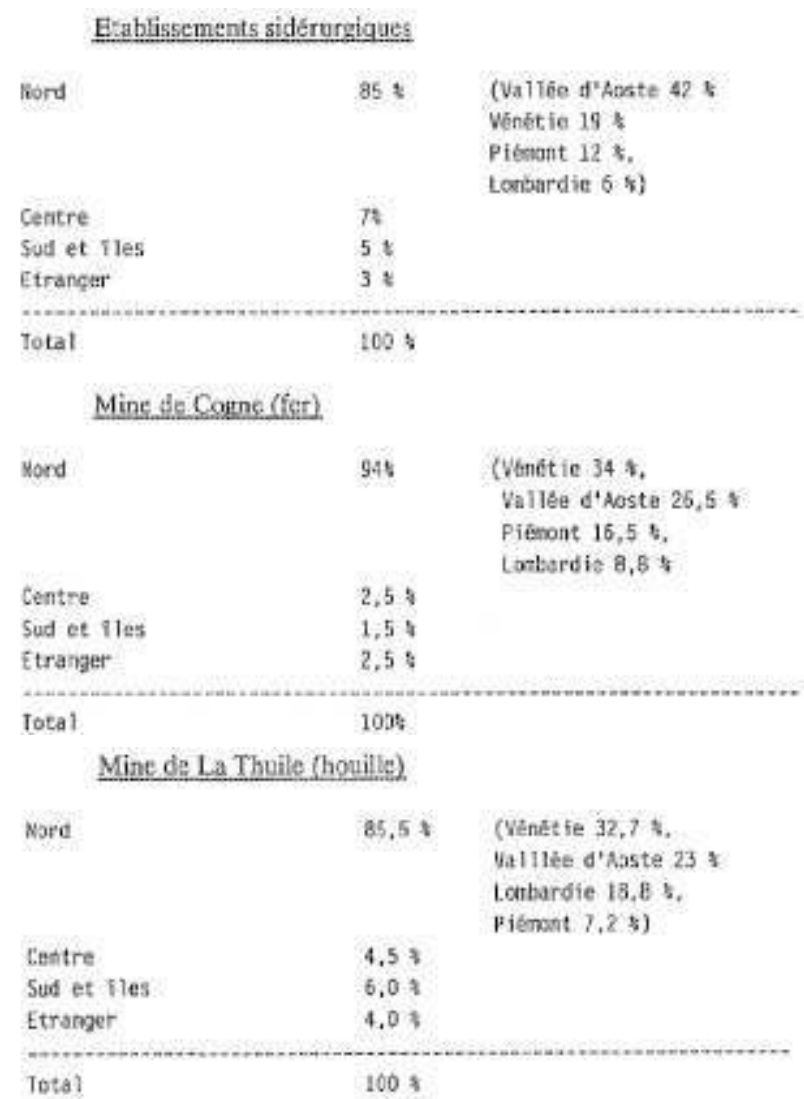

En général on peut constater que la composante locale, c'est-à-dire valdotaine, est largement majoritaire dans les établissements sidérurgiques, tandis que dans les secteurs miniers ce groupe ne dépasse jamais $25 \%$ du total de la main d'œuvre.

Compte tenu des conditions de travail particulièrement pénibles dans les mines, on peut supposer une disposition plus grande à l'adaptation parmi ceux qui ont dû émigrer afin de trouver du travail. Mais en regardant de plus près, grâce aux dossiers individuels, on s'aperçoit que les mineurs à proprement parler sont en large majorité originaires des régions qui ont, parfois depuis longtemps, une tradition minière. Une analyse plus fine des provenances par commune indique en fait que les bassins de recrutement sont relativement définis, et correspondent en même temps à des aires de tradition historique du travail minier, ou encore à des milieux où des liens directs entre entreprise et communauté locale se sont établis afin d'assurer un flux continu de main d'oeuvre; d'autres aires encore peuvent être définies comme des bassins de recrutement de vastes proportions et à qualification relativement faible, telles la plaine vénitienne (provinces de Vérone, Padoue et Vicence) ${ }^{62}$. Cette dernière composante est particulièrement importante dans les établissements sidérurgiques, tandis que les deux premières forment le gros des travailleurs du secteur minier, où l'on trouve par centaines des natifs des communes préalpines de la province de Bellune ${ }^{63}$.

Cette situation est confirmée par les données de la Lombardie et du Piémont. Les mineurs de ces régions viennent en grande partie des aires de tradition minière, comme la partie alpine des provinces de Bergame et Brescia ${ }^{64}$, ou des communes de Traversella et Brosso, 
lieux historiques de l'activité minière piémontaise du XIX siècle. Ces mêmes régions fournissent également de la main d'oeuvre au secteur sidérurgique, mais dans ce cas il s'agit d'ouvriers qui viennent pour la plupart des villes, Turin, Novare ou Milan.

Le secteur sidérurgique, plus généralement, pose à la fois des problèmes de qualité et de quantité. Si l'on retrouve ici par milliers des ouvriers provenant des zones qui ont connu une émigration massive vers les pôles industriels de toute l'Europe du nord (Allemagne, France et Belgique en particulier), il est évident, surtout au début des années vingt, qu'un groupe constitué surtout d'une main d'oeuvre spécialisée a été recruté à Gênes, Milan, Terni ou Piombino, dans les régions de travail sidérurgique, afin de constituer le noyau ouvrier de l'entreprise. La composante méridionale ne représente qu'un faible pourcentage, en légère hausse pendant la deuxième guerre mondiale; il s'agit principalement de Calabrais issus des communes de San Giorgio Morgeto, Melito Porto Salvo, Montebello Ionico et Polistena; les rares Siciliens viennent de Bronte et de l'arrière-pays catanais. Un dernier aperçu, enfin, de la composante constituée par les étrangers, qui sont en réalité des Italiens émigrés de retour. Il s'agit pour la plupart d'émigrés de la seconde génération, qui sont nés principalement en France, Belgique et Suisse ; il n'est quand même pas rare de rencontrer des travailleurs provenant des EtatsUnis, du Brésil et d'Argentine.

51 On peut aborder maintenant le problème de la qualification ouvrière à l'aide des données suivantes :

Tableau n $^{\circ} 3$ (niveau de qualification des ouvriers sidérurgiques) ${ }^{65}$

\begin{tabular}{|c|c|c|c|}
\hline & 1922 & $193 \%$ & $\| 9 \% ?$ \\
\hline Três fatble & $6 ? ?^{2}$ & $25: 5$ & $27 \div$ \\
\hline Faible & 1.2: & $38{ }^{3}$ & $22:$ \\
\hline Moyenrme? & $23^{6}$ & $18: 8$ & $25: 5$ \\
\hline Haute & $7 \%$ & $1.0: 4$ & 8 \\
\hline Très hatute & $7 \%$ & $10 \%$ & $8+2$ \\
\hline
\end{tabular}

Tableau $n^{\circ} 4$ (données relatives au secteur minier)

\begin{tabular}{|c|c|c|c|}
\hline & 1922 & 1932 & $.194 ?$ \\
\hline Très fatble & $56 \%$ & $41 \%$ & $42 \%$ \\
\hline Faible & $6 \%$ & .14. $\%$ & $29 \div$ \\
\hline Moyennา & $26:$ & $27 \div$ & 1178 \\
\hline Haute & $9:$ & $9:$ & $16 \%$ \\
\hline Tress haute & $3:$ & $9 \%$ & $6 \%$ \\
\hline
\end{tabular}

Pour obtenir ces données, un travail préliminaire complexe a été nécessaire; en effet, dans les « livres d'embauche », est mentionnée la fonction et non pas la qualification, et 
ceci au moment de l'entrée à l'usine; nous ne tenons donc pas compte du parcours professionnel des travailleurs.

On a donc recensé les 149 tâches de notre échantillon, et pour évaluer ces postes de travail on a utilisé en premier un «tableau des salaires par poste ${ }^{66}$, dont les informations ont été intégrées et corrigées à l'aide d'interviews de plusieurs anciens travailleurs de l'entreprise. L'ensemble de cette opération a donné lieu à un «Tableau de conversion des fonctions en un classement unique $\aleph^{67}$, sur la base duquel on a attribué une valeur numérique à chacun des postes de travail, ce qui en définitive a permis le traitement sur ordinateur de ces données ${ }^{68}$.

Les données des tableaux $n^{\circ} 3$ et $n^{\circ} 4$ en sont un premier exemple. On peut entrevoir, sur la longue durée, une tendance à la compression des couches à "faible » et «très faible " qualification, face au passage des couches à « haute » et "très haute » qualification de $14 \%$ à $26 \%$ entre 1922 et 1942. Il s'agit en effet d'un indicateur assez intéressant. Le processus général de rationalisation de l'organisation du travail, qui caractérise le début des années trente, ferait penser au contraire qu'une tendance à la massification du travail à faible ou très faible qualification soit plus clairement visible. En fait, cette réalité est peut-être visible au niveau de la qualification "moyenne », qui ne cesse de s'accroître, passant de $12 \%$ en 1922 à $18 \%$ en 1932 et $25 \%$ en 1942 ; mais malgré cela le problème reste entier. Une explication devrait probablement être recherchée dans les transformations profondes de l'usine. On constaterait qu'une attention particulière est accordée à l'organisation du travail, comme le montre l'étude de Fossati de $1927^{69}$; qu'une massification du travail est prévisible dans une entreprise qui passe en vingt ans de 750 à 6.200 ouvriers; de plus le recul de la «professionnalité ", tout particulièrement dans les aciéries avec l'introduction progressive de la chimie, ne peut que faire légitimement prévoir un gonflement de la couche inférieure et moyenne des travailleurs; c'est pourquoi l'explication de cette évolution est à chercher probablement plus dans les modifications de l'organisation de la production que dans celles de l'organisation du travail.

En d'autres termes, l'entreprise passe en vingt ans de la production de fonte dans les hauts fourneaux électriques, de quelques aciers spéciaux et d'un petit laminoir, à une organisation très complexe, où à côté de la production de fonte et d'acier, intervient une diversification progressive de la production : aciers spéciaux, alliages, pièces forgées aux formes les plus différentes et pour les marchés les plus divers : marine militaire, aviation, transports ferroviaires, etc. Soit, en même temps, une spécialisation dans la production des matières premières, mais aussi une verticalisation de la production qui conduira l'entreprise à la production sur place de torpilles, de canons et autres produits finis, surtout dans le secteur des armements.

56 C'est donc probablement là que se trouve une explication au moins partielle du phénomène, étant donné que cette diversification a sûrement entraîné la présence toujours plus importante d'un noyau ouvrier de métier à haute qualification, qui était la structure porteuse d'un système de production si différencié.

57 Le dernier groupe de données concerne la mobilité professionnelle des ouvriers. 
Tableau $n^{\circ} 5$ (durée des rapports de travail calculée sur la moyenne des cinq années de chaque période) $)^{70}$

\begin{tabular}{lllll} 
& 1 & 2 & 3 & 4 \\
\hline $1917 / 21$ & & 22,22 & 69,96 & 14,81 \\
\hline $1922 / 26$ & 31,43 & 48,57 & 11,43 & 8,57 \\
\hline $1927 / 31$ & 14,90 & 40,73 & 21,85 & 22,52 \\
\hline $1932 / 36$ & 11,20 & 21,99 & 31,12 & 35,68 \\
\hline $1937 / 41$ & 12,66 & 34,37 & 29,97 & 23,00 \\
\hline $1942 / 46$ & 12,71 & 32,24 & 22,59 & 32,47 \\
\hline $1947 / 52$ & 9,92 & 18,18 & 14,05 & 57,85
\end{tabular}

Ce qui ressort de ces données, comme dans d'autres travaux récents ${ }^{71}$, c'est le très haut turn-over des forces de travail pendant l'entre-deux guerres. Mobilité qu'à travers l'analyse des dossiers individuels on peut qualifier de "volontaire », dans la mesure où il ne s'agit que rarement d'autres facteurs tels que licenciement, service militaire, passage au niveau d'employé, retraite ou décès. Ceci est particulièrement net si l'on regarde dans quelle mesure le deuxième après-guerre représente une rupture, un profond changement de direction. Autour d'un noyau fort et stable, ni particulièrement fourni, ni particulièrement composé de la partie supérieure de la professionnalité ouvrière, évolue une masse constituée de travailleurs qui entrent et sortent plusieurs fois, sans préjudice d'un séjour temporaire dans d'autres entreprises ou dans d'autres secteurs, particulièrement l'agriculture ou le bâtiment.

Le tableau montre clairement ce phénomène. En 1917, à la constitution du noyau fondamental du corps ouvrier, les classes (3) et (4) représentent à elle seules 77,77 \% du total. En 1922 et 1927, l'expansion de l'emploi en direction des travailleurs à moyenne et faible qualification correspond à un bond en avant du groupe ouvrier, dont la présence à l'usine ne dépasse pas les 500 jours; comme on le voit les classes (1) et (2) représentent $80 \%$ en 1922 et $55,63 \%$ en 1927. Pendant la crise du début des années trente, le corps le plus solide et le plus stable se maintient; en effet les groupes (3) et (4) reprennent l'avantage. En 1937, avec la reprise de la production liée à l'aventure coloniale, c'est au contraire, de nouveau, la partie basse qui enregistre le chiffre le plus haut. Pour conclure, si l'on sait que le second après-guerre représente un tournant au niveau de la stabilité de l'emploi, on ne s'attendrait pas à ce changement si rapide entre 1947 et 1951: à la première place on trouve, avec $57,85 \%$, les travailleurs qui demeurent au moins 2.000 jours dans l'usine. 


\section{Conclusions}

60 Ce deuxième aspect, par la richesse des données, la précision avec laquelle est mis en évidence un grand nombre de questions, pourrait faire l'objet d'une réflexion bien plus vaste dont on pourrait imaginer qu'elle se développe autour du croisement de différents éléments, par exemple : qualification/mobilité, mobilité/provenance géographique, etc.

Pour le moment, par ce travail sur ces données, qui est encore fort incomplet, on a voulu alimenter le débat, qui est aussi un débat politique et culturel ; apporter un exemple qui permette également de comparer les conditions matérielles de développement d'une entreprise et d'une classe ouvrière, loin des grandes métropoles du nord de l'Italie, pour apporter en dernière analyse un élément de plus dans la compréhension de la complexité territoriale de l'essor industriel au sud des Alpes.

\section{NOTES}

1. Emanuele Gazzo, I cent'anni dell'Ansaldo, Gênes, 1953.

2. Franco Bonelli, Lo sviluppo di una grande impresa in Italia: la Terni dal 1884 al 1962, Turin, 1975, p. 16 (au départ de son programme sidérurgique l'Ansaldo avait utilisé l'aide technologique d'entreprises anglaises et du groupe allemand Krupp) ; voir aussi : Storia d'Italia Einaudi, vol. 4, t. 1 (par V. Castronovo), p. 162..

3. Le capital social de l'Ansaldo passe de 30 millions en 1916 à 500 millions en 1918.

4. Valerio Castronovo, L'industria italiana dall' ottocento ad oggi, Milan, 1980.

5. Antonia Carparelli, «La siderurgia nel periodo tra le due guerre » dans C. Binel, Dall' Ansaldo alla Cogne, Milan, 1985 ; et Franco Bonelli, Acciaio per l'industrializzazione, Torino, Einaudi, 1982.

6. Giorgio Mori, «Le guerre parallele. L'industria elettrica in Italia nel periodo della Grande Guerra », Studi Storici, n 2, 1973 ; et Bruno Bezza, Energia e sviluppo. L'industra elettrica italiana e la Società Edison, Torino, Einaudi, 1986.

7. Pour quelques informations sur l'entreprise Ansaldo, voir note suivante.

8. Archives Nationales de Paris, $F^{12} 7260$, Rapport du Consulat de France à Turin du 27 janvier 1919 : «Avant la guerre de 1914, d'après les renseignements qui m'ont été fournis par la Chambre de Commerce française de Turin, elle (l'Ansaldo) exploitait les usines suivantes: Établissement Mécanique (dit «meccanico») (Sampierdarena-Gênes), Fabrique de Locomotives (Sampierdarena-Gênes), Aciérie de Cornigliano Campi et section blindages Marrel (CorniglianoGênes), Usine électronique (Cornigliano-Gênes), Usine Delta (Cornigliano-Gênes), Chantier naval pour cuirassés de Sestri Ponente (Gênes), Chantier d'armement naval du port de Gênes (...). Depuis la guerre les sections suivantes se sont ajoutées: Usine de construction d'artillerie (Sampierdarena-Gênes), agrandissement formidable des aciéries de Cornigliano, fabrique de munitions de la Fiumara (Gênes), usine pour la construction de moteurs à explosion, usine pour la production de l'oxygène et de l'hydrogène, nouvelle section des usines d'artillerie, fonderie de bronze et autres métaux, fours électriques pour la production des ferro-alliages, chantier naval Savoia, usine de construction de moteurs pour l'aviation, usine et chantiers FIAT-San Giorgio de Turin et La Spezia, 4 usines de construction d'avions de guerre dont la S.I.T. et Pomilio de Turin, 
fabrique de tubes en acier, usine pour la fabrication des douilles d'artillerie, chantier naval de Sestri pour la construction de cargo-boats en bois, section spéciale pour les matériaux réfractaires, mines de fer de Cogne (Vallée d'Aoste), usines de ferros et aluminium de Villeneuve (Vallée d'Aoste), aciérie électrique d'Aoste en construction, qui pourra occuper plus de 6.000 ouvriers, nouvelle usine de projectiles de Sestri, achat des usines Fossati (Sestri), achat de l'affaire Talamona à Turin, fabrique de moteurs d'aviation du type Isotta-Fraschini... ».

9. E. Gazzo, I cent' anni dell' Ansaldo, op. cit. et Paride Rugafiori, Uomini, macchine e capitali, Milan, 1981.

10. Corrado Binel, Dall' Ansaldo alla Cogne, Milan, 1985.

11. V. Rocca, « Il sindacato del ferro in Italia », La Riforma Sociale, $\mathrm{n}^{\circ}$ VI, 1899 ; et E. Giretti, « La Società Terni, il governo e il Trust metallurgico ", Il Giornale degli Economisti, n XVI, 1903.

12. Archivio DeltaCogne cité désormais ADC), acte constitutif. Capital 3.500 .000 lires. Actionnaires fondateurs de la société : Comte Charles Van der Straten-Ponthoz (né à Ponthoz, Belgique); Alfred Theys (né à Lodelinsart, Belgique) ; Raffaele Bombrini (né à Gênes); Chierici Giorgio (né à Porto Maggiore de Ferrara); Armando Raggio (né à Gênes) et Giuseppe Cenni (né à Gênes) représentants de la "Società Alti Forni e Fonderie di Piombino "; Cesare Ruffinoni (né à Bordogna) ; Giuseppe Ferro (né à Gênes); Rodolfo Barabino (né à Gênes); Emilio Romano (né à Gênes); Carlo Alberto Bombrini (né à Gênes); Federico Ricci (né à Gênes); Emile Foltzer (né à Fontaine-le-Bourg) ; Filippo Tassara (né à Voltri) ; Dimitris Rodocanachi (né à Galatz, Roumanie) ; Giuseppe Lottero (né à Gênes); Charles Mac Nevin (né à Liverpool, Angleterre); Giuseppe Colano (né à Gênes).

13. Archivio Storico Ansaldo (ASA) 591/37, lettre de François Tardy à Mario Perrone : « M. Alfred Theys, mort à Bruxelles au commencement de la guerre, et peu de temps après avoir cédé ses actions de la Cogne (mine, ndr.) dont il était on peut dire le fondateur ».

14. P. Farinet, I Precursori, Aoste, 1922.

15. Archives Nationales de Paris, $\mathrm{F}^{14} 1034,1035,1036$.

16. ASA 91/46, relation de Leotardi à Perrone, 20 octobre 1916.

17. F. Farinet, Nos forces motrices, Aoste, 1895 ; et Lucat et Brocherel, «Aménagement des forces hydrauliques du Lys ", Augusta Praetoria, 1921.

18. Société sidérurgique fondée à Gênes en 1905 (ILVA, nom latin de l'île d'Elbe).

19. Valerio Castronovo, Giovanni Agnelli,Turin, Einaudi, 1977, p. 235.

20. Groupe sidérurgique situé dans la ville de Terni et fondé par Stefano Breda le 10 mars 1884.

21. Attilio Odero: industriel propriétaire des chantiers de constructions navales de Sestri (Gênes) et fondateur du groupe Odero-Terni-Orlando (O.T.O.), par incorporation de la société de constructions navales Orlando de Livourne et de la société Odero-Terni.

22. Arturo Bocciardo : administrateur délégué des aciéries Terni à partir d'avril 1921.

23. Valerio Castronovo, L'industria italiana dall ottocento ad oggi, Milan, 1980.

24. Valerio Castronovo, Giovanni Agnelli, pp. 233-34.

25. Archivio della Banca d'Italia (ABI), $\mathrm{n}^{\circ} 25$ (445-463 : «Presidenza Soc. gruppo Ansaldo ») : lettre de Stringher au Ministre Facta, 25 mars 1922.

26. Paride Rugafiori, Uomini, macchine e capitali, Milan, 1981, p. 32.

27. Réunions du gouvernement des 17 et 21 février 1922, qui donneront lieu à l'Arrêté Royal du 14 juin 1923, nº 1475 (Gazzetta ufficiale, 17 juillet 1923).

28. Ernesto Rossi, Padroni del vapore e fascismo, Bari, 1966 ; et Paride Rugafiori, « Cofindustria », in Levi, Levra, Tranfaglia, Storia d'Italia, Firenze, 1978, vol. 1.

29. Louis Maillard, Paul Girod 1878-1951, Paris, 1952.

30. Journal du four électrique, $\mathrm{n}^{\circ} 3-1922, \mathrm{n}^{\circ} 10-1922, \mathrm{n}^{\circ} 11-1922, \mathrm{n}^{\circ} 1-1971, \mathrm{n}^{\circ} 7-1971, \mathrm{n}^{\circ}$ 8-1971; voir aussi Raoul Blanchard, «L'électro-métallurgie et l'électro-chimie dans les Alpes françaises ", Revue de Géographie alpine, Grenoble, 1924 ; et Broise, Demotz, Devos, Bon, Histoire d'Ugine, Annecy, 1975. 
31. A la suite de deux augmentations, le capital social en 1926 est composé comme suit : AnsaldoCogne (35 millions), Paul Girod (2,5 millions), Banque Suisse (4,3 millions), Jules Block (2,5 millions), Louis Vaucher ( 0,1 million) et Eugène Borel ( 0,6 million).

32. Eugenio Fossati, Lavore produzione in Italia, Turin, 1951, p. 507.

33. Sur le problème de l'industrie italienne dans la crise de 1929, Valerio Castronovo, L'industia italiana dall ottocento a oggi, Milan, 1980, pp. 188 sqq.

34. Archivio Agostino Rocca auprès de la Fondazione Luigi Einaudi de Turin (cité désormais AAR) : Revisione « Cogne » e « Aosta », 1933/1934.

35. Archivo Storico dell'Istituto per la Ricostruzione Industriale (ASIRI) : rapport de l'ingénieur Carli, "Sull'industria siderurgica in Italia con particolore riguardo all'ILVA », fas. Siderurgica I, 1933.

36. Traduction de "siderurgia bellica speciale ", terme sous lequel on désigne à cette époque ce noyau d'entreprises productrices d'aciers spéciaux, d'alliages, etc., susceptibles d'être utilisés dans l'industrie de guerre.

37. Gianni Toniolo (édit.) : Industria e banca nella grande crisi (1929-1934), Milan, 1978.

38. Franco Bonelli, La sviluppo di una grande impresa in Italia : la Terni 1884-1962, Turin, 1975, p. 212.

39. ASIRI : Rapport général sur Cogne et Aosta, Siderurgia I, 31 mars 1934, p. 85.

40. «L'economia italiana nel sessenio 1931-1936», dans Banca d'Italia, IIe partie, vol. II ; et aussi AAR. "Siderurgia bellica»: a) sur Cogne et Aosta, 30 mars 1934; b) sur l'Ansaldo SA, 26 juillet 1933 ; c) notes sur la Cogne présentées au comité technique pour la « Siderurgia bellica speciale ", 30 juin 1934 ; d) notes sur l'usine Ansaldo, 11 juillet 1934 ; e) conclusion du même comité, 10 août 1934 ; voir aussi, toujours dans AAR.35.6, "Nuova società per il rilievo e l'esercizio delle acciaierie di Aosta e Cornigliano : elementi per la sua impostazione patrimoniale e finanziaria », 5 septembre 1934.

41. AAR., Lettre d'Agostino Rocca à Donato Menichella, 31 janvier 1935.

42. Alliage de carbure de tungstène et cobalt ou nickel obtenu par frittage. Il est utilisé principalement dans la fabrication d'outillages.

43. Ibid.

44. Archivio Storico Autonoma Valle d'Aosta (ASVDA), fasc. 8.

45. AAR ; 35.16, " Premiers éléments pour une convention entre la S.I.A.C.C. et la Terni » (s.d., mais 1935).

46. Voir le discours de Benito Mussolini du 23 mars 1936 à la deuxième assemblée nationale des corporations. Texte publié dans B. Mussolini, Opera omnia, par D. et E. Susmel, Florence, 1959, vol. XXVII, pp. 241-248.

47. Sur l'activité de la Cogne pendant la deuxième moitié des années trente, on n'a pu consulter que les rapports du Conseil d'administration de l'entreprise, qui d'ailleurs sont particulièrement succints.

48. Victorio Valletta : dirigeant du groupe FIAT de tout premier plan dès les années 20.

49. Archivio Thaon de Revel ; 31.1.38 ; « lettre de l'ingénieur colonel Bettica », 5 décembre 1942.

50. Archivio Thaon de Revel : lettre de Bettica en date du 21 décembre 1942.

51. ibidem (Courgné : ville de la haute province de Turin)

52. Ettore Passerin d'Entrèves (éd.) : Guerra e resistenza nelle regioni alpine occidentali 1940-45, Milan, 1980 ; en particulier l'essai de G. Perrona.

53. Pendant notre recherche nous avons eu accès à une partie seulement des archives; d'ailleurs, c'est précisément pendant notre travail que l'on a retrouvé dans le sous-sol de l'usine environ 500 cartons de documents non inventoriés, qui concernent en particulier les années trente.

54. «Libri matricola » est traduit par « livres d'embauche »; il s'agit des registres dans lesquels on a consigné les ouvriers embauchés et licenciés par ordre chronologique. On y trouve : nom, prénom, date et lieu de naissance, date d'embauche, date de licenciement, qualification au 
moment de l'embauche (par qualification, on entend plutôt la «tâche » que la qualification à proprement parler).

55. Pour une première approche de l'histoire de la classe ouvrière italienne pendant le fascisme, voir : Annali Fondazione Giangiacomo Feltrinelli ; La classe operaia durante il fascismo, Milan, 1981.

56. De 1917 au mois de décembre 1945.

57. Voir la Revue Quaderni Rossi ; et aussi Romano Alquati, Sulla FIAT e altri scritti, Milano, 1975.

58. En particulier les travaux de Luisa Passerini, Torino operaia e fascismo, Roma, 1984 ; Maurizio Gribaudi, Mondo operaio e mito operaio, spazi e percorsi sociali a Torino nel primo novecento, Torino, 1987.

59. Par exemple: F. Piva et G. Tattara (éd.), I primi operai di Marghera, Venise, 1983 ; Paride Rugafiori, Uomini, macchine et capitali: l'Ansaldo durante il fascismo (1922-1945), Milano, 1981; Cristina Biffoli et Michele Lungonelli, «Una classe operaia in formazione: i siderurgici di Portoferraio, 1901-1905 », Studi Storici, n 1, 1985 ; et R. Prister, Anna Luperini, Andrea Preve, «Personale, rapporti di lavoro e nuovi servizi nella U.I.T.E. 1895-1940», Quaderni delle'Archivio Storico Azienda Municipalizzata Trasporti di Genova, $\mathrm{n}^{\circ}$ 1, 1986.

60. Voir par exemple Franco Ramella, Terra e telai, Turin, 1984.

61. L'ensemble des données a été traité sur l'ordinateur du «C.S.I. Piemonte » de Turin.

62. Les communes que l'on retrouve le plus fréquemment sont: Lonigo, Sarego, Gambellara, Lusiana, Cervarese et Pressana.

63. Par exemple, les communes de Mel, Lamon, Sospirolo et Agordo.

64. En particulier les communes de Leffe, Pisone et Breno.

65. On a réparti les données en 5 niveaux de qualification, ce qui correspond aux 5 niveaux de la qualification ouvrière de l'inquadramento unico (voir à ce sujet la note 55).

66. «Tavola delle paghe di posto », traduit en français « Tableau des salaires par poste "; il s'agit d'une sorte de manuel qui établit la rémunération horaire pour chaque poste de travail. Sur ce document, qui existait pendant les années trente, ne se retrouve pas la totalité de nos 149 postes de travail.

67. "Inquadramento unico ", traduit en français "Classement unique », contrat de travail des ouvriers métallurgistes des entreprises privées du 19 avril 1973, qui définit un système de qualification unique des ouvriers et des employés. Pour le débat préliminaire, voir Antonio Lettieri, «Inquadramento unico e strategia della qualificazione », Quaderni sindacali, n $35,1971$. Tableau de conversions des fonctions en classement unique: fonction (italien), classement unique $\left(\mathrm{n}^{\circ}\right)$, fonction (français).

68. Un travail similaire a été réalisé par des chercheurs turinois à la Soc. Naz. delle Officine di Savigliano. Voir Anna Donvito et Gianni Garbarini, «Senz'altra formalità che il reciproco preavviso : le officine di Savigliano, 1904-1914», Italia contemporanea, décembre 1984, fasc. 157.

69. M. Fossati : "L'organisation scientifique du travail dans les mines et les usines électrosidérurgiques Ansaldo-Cogne", rapport présenté à la conférence sur l'organisation scientifique du travail de Bruxelles en 1927.

70. Les données sont divisées en 4 colonnes : dans la première, en pourcentage, le groupe ouvrier qui a travaillé de zéro à 60 jours ; dans la colonne 2, ceux qui ont travaillé de 61 à 500 jours ; dans la colonne 3, ceux qui ont travaillé de 501 à 2.000 jours et enfin, dans la colonne 4 , ceux qui ont travaillé plus de 2.000 jours. Les ouvriers qui ont été embauchés plusieurs fois apparaissent une seule fois, et leur présence à l'usine n'est pas cumulée.

71. Piva et Tattara, Primi operai di Marghera, Venise, 1983 ; voir également note 59. 


\section{AUTEUR}

\section{CORRADO BINEL}

Corrado BINEL appartient à une famille d'Aoste, est architecte, diplômé de l'Istituto Politecnico de Turin, et docteur de 3ème Cycle en Histoire de l'E.H.E.S.S. depuis 1985 (intitulé de la thèse : La sidérurgie du Val d'Aoste aux XIX et XX ${ }^{e}$ siècles : l'Ansaldo à Cogne). 